1 Table S1 The input of organic compounds for MCM model $\left(\mathrm{pmol} \mathrm{mol}^{-1}\right)^{\mathrm{a}}$

\begin{tabular}{|c|c|c|}
\hline Species & $\mathrm{PM}_{2.5}<75 \mu \mathrm{g} \mathrm{m}^{-3}$ & $\mathrm{PM}_{2.5} \geq 75 \mu \mathrm{g} \mathrm{m}^{-3}$ \\
\hline Methane & 1900 & 1900 \\
\hline Formaldehyde & 3.49 & 7.65 \\
\hline Acetaldehyde & 2.36 & 5.89 \\
\hline Ethane & 3.675 & 10.34 \\
\hline Propane & 2.165 & 6.93 \\
\hline isobutane & 0.815 & 2.52 \\
\hline n-Butane & 1.135 & 3.58 \\
\hline isopentane & 0.65 & 2.26 \\
\hline n-Pentane & 0.465 & 1.51 \\
\hline 2-methylpentane & 0.235 & 0.77 \\
\hline n-Hexane & 0.265 & 0.84 \\
\hline Ethylene & 2.245 & 6.64 \\
\hline Propene & 0.505 & 1.19 \\
\hline 1-Butene & 0.14 & 0.36 \\
\hline isoprene & 0.085 & 0.12 \\
\hline Acetylene & 1.945 & 7.82 \\
\hline Benzene & 0.63 & 1.95 \\
\hline Toluene & 0.925 & 2.84 \\
\hline Ethylbenzene & 0.295 & 1.08 \\
\hline m/p-Xylene & 0.605 & 2.03 \\
\hline o-Xylene & 0.175 & 0.63 \\
\hline Acrolein & 0.1 & 0.25 \\
\hline Propanal & 0.31 & 0.66 \\
\hline Acetone & 2.41 & 6 \\
\hline MTBE & 0.2 & 0.71 \\
\hline Methylvinylketone & 0.095 & 0.32 \\
\hline Methylethylketone & 0.435 & 1.41 \\
\hline n-Hexanal & 0.18 & 0.48 \\
\hline Chloromethane & 0.32 & 0.91 \\
\hline Dichloromethane & 1.895 & 4.4 \\
\hline Chloroform & 0.395 & 1.31 \\
\hline 1,2-Dichloroethane & 0.5 & 1.91 \\
\hline 1,2-Dichloropropane & 0.35 & 1.46 \\
\hline 1,1,2,2-Tetrachloroethane & 0.315 & 0.32 \\
\hline
\end{tabular}

$2{ }^{a}$ The mixing ratio of methane is from Wang et al. (2001). The mixing ratios of formaldehyde and

3 acetaldehyde are from Rao et al. (2016). The mixing ratios of other organic compounds are from Wu et

4 al. (2016)

\title{
5 Reference
}

6 Rao, Z., Chen, Z., Liang, H., Huang, L., and Huang, D.: Carbonyl compounds over urban Beijing: 
9 Wang, Y., Zhou, L., Wang, M., and Zheng, X.: Trends of atmospheric methane in Beijing, $10 \quad$ Chemosphere, 3, 65-71, 2001.

11 Wu, R., Li, J., Hao, Y., Li, Y., Zeng, L., and Xie, S.: Evolution process and sources of ambient volatile organic compounds during a severe haze event in Beijing, China, Sci. Total Environ., 560, 62-72,

13 2016. 